Mutations in Myelodysplastic Syndromes. N Engl I Med. 2011;364(26):2496-2506.

3. Hunter AM, Sallman DA. Current status and new treatment approaches in TP53 mutated AML. Best Pract Res Clin Haematol. 2019;32(2):134-144.

4. Sallman DA, Komrokji R, Vaupel C, et al. Impact of TP53 mutation variant allele frequency on phenotype and outcomes in myelodysplastic syndromes. Leukemia. 2016;30(3):666-673.

5. Haase D, Stevenson KE, Neuberg D, et al. TP53 mutation status divides myelodysplastic syndromes with complex karyotypes into distinct prognostic subgroups. Leukemia. 2019;33(7):1747-1758

6. Montalban-Bravo G, Kanagal-Shamanna R, Benton CB, et al. Genomic context and TP53 allele frequency define clinical outcomes in TP53-mutated myelodysplastic syndromes. Blood Adv. 2020;4(3):482-495

7. Bernard E, Nannya Y, Yoshizato T, et al. TP53 State Dictates Genome Stability, Clinical Presentation and Outcomes in Myelodysplastic Syndromes. Blood. 2019;134(Supplement_1):675675

8. Hunter AM, Sallman DA. Targeting TP53 Mutations in Myelodysplastic Syndromes. Hematol Oncol Clin North Am. 2020;34(2):421-440.

9. Sallman DA, DeZern AE, Garcia-Manero G, et al. Phase 2 Results of APR-246 and Azacitidine (AZA) in Patients with TP53 mutant Myelodysplastic Syndromes (MDS) and Oligoblastic Acute Myeloid Leukemia (AML). Blood. 2019;134(Supplement_1):676-676.

10. Cluzeau T, Sebert M, Rahmé R, et al. APR-246 Combined with Azacitidine (AZA) in TP53 Mutated Myelodysplastic Syndrome (MDS) and Acute Myeloid Leukemia (AML). a Phase 2 Study By the Groupe Francophone Des Myélodysplasies (GFM). Blood. 2019;134(Supplement_1):677-677.
11. Maslah N, Salomao N, Drevon L, et al. Synergistic effects of PRIMA1Met (APR-246) and Azacitidine in TP53-mutated myelodysplastic syndromes and acute myeloid leukemia. Haematologica. 2019; xxx

12. Boettcher S, Miller PG, Sharma R, et al. A dominant-negative effect drives selection of TP53 missense mutations in myeloid malignancies. Science. 2019;365(6453):599-604.

13. Lambert IM, Gorzov P, Veprintsev DB, et al. PRIMA-1 reactivates mutant p53 by covalent binding to the core domain. Cancer Cell. 2009;15(5):376-388.

14. Zhang Q, Bykov VJN, Wiman KG, Zawacka-Pankau J. APR-246 reactivates mutant p53 by targeting cysteines 124 and 277. Cell Death Dis. 2018;9(5):439.

15. Lehmann S, Bykov VI, Ali D, et al. Targeting p53 in vivo: a first-inhuman study with p53-targeting compound APR-246 in refractory hematologic malignancies and prostate cancer. J Clin Oncol 2012:30(29):3633-3639.

16. Deneberg S, Cherif H, Lazarevic V, et al. An open-label phase I dosefinding study of APR-246 in hematological malignancies. Blood Cancer J. 2016;6(7):e447.

17. Cluzeau T, Dubois A, Jacquel A, et al. Phenotypic and genotypic characterization of azacitidine-sensitive and resistant SKM1 myeloid cell lines. Oncotarget. 2014;5(12):4384-4391.

18. Bykov VJ, Zhang Q, Zhang M, Ceder S, Abrahmsen L, Wiman KG Targeting of Mutant p53 and the Cellular Redox Balance by APR-246 as a Strategy for Efficient Cancer Therapy. Front Oncol. 2016;6:21

19. Peng X, Zhang MQ, Conserva F, et al. APR-246/PRIMA-1MET inhibits thioredoxin reductase 1 and converts the enzyme to a dedicated NADPH oxidase. Cell Death Dis. 2013;4(10):e881.

20. Demir S, Boldrin E, Sun Q, et al. Therapeutic targeting of mutant p53 in pediatric acute lymphoblastic leukemia. Haematologica. 2020;105(1):170-181.

\title{
Peripheral T-cell lymphoma diagnosis: building a molecular tool
}

\section{Miguel A Piris ${ }^{1,2}$}

${ }^{1}$ Department of Pathology, Hospital Universitario, Fundación Jiménez Díaz and ${ }^{2} C I B E R O N C$, Madrid, Spain

E-mail: MIGUEL A PIRIS - mapirispinilla@gmail.com

doi:10.3324/haematol.2020.249052

T -cell lymphoma (TCL) has quite a poor probability of survival (around $25-30 \%$ of patients after 5 years), which contrasts with the progress that has recently been made in Hodgkin lymphoma and B-cell lymphoma. ${ }^{1-3}$ Some recently defined TCL types, such as anaplastic large-cell lymphoma (ALCL), have a better clinical outcome. However, the majority of cases diagnosed with peripheral T-cell lymphoma (PTCL) will eventually die of the disease, and in some specific tumor types, such as intestinal TCL, the prognosis is even more miserable.

Poor survival probability in this context is associated with serious difficulties in lymphoma diagnosis when using routine morphological and immunohistochemistry tools. PTCL classification involves division into multiple subtypes, typically of low frequency and with hazy distinctions (Figure 1). As a consequence, different studies coincide in achieving a very low rate of reproducibility in TCL diagnosis, especially in recognizing ALK-negative ALCL, and distinguishing between PTCL-not otherwise specified (NOS) and PTCL with TFH phenotype or angioimmunoblastic TCL. ${ }^{4}$

An important feature of this situation is that the relative frequencies of the tumor types are quite low, which makes it difficult to design and develop clinical trials, and this hampers the introduction of new drugs for PTCL therapy.
Nevertheless, these difficulties have inspired some research groups to provide essential information about the molecular basis of TCL pathogenesis, and to identify some attractive and challenging therapeutic targets..$^{5.9}$

Drieux and co-workers, ${ }^{10}$ in a joint project involving French, Belgian and Swiss hospitals, are now addressing the radical proposal that molecular diagnosis may give a more precise and reproducible way of classifying TCL cases. Using a technique applicable to paraffin-embedded tissue, they measure the expression of 20 genes, including 17 markers relevant to T-cell classification, one EpsteinBarr virus-related transcript, and frequently mutated variants of RHOA (G17V) and IDH2 (R172K/T). Selected genes allow the identification of several entities: TFH cells, the normal counterparts of angioimmunoblastic TCL; TH1 and TH2 phenotypes, which reflect the diversity of PTCL-NOS; T-regulatory cells, for distinguishing ATLL; the cytoxic markers, CD30 and ALK, for identifying ALCL; and CD56 and EBER1, to discriminate T/natural killer (NK)-cell lymphomas.

The results validate the solid basis of the currently used PTCL classification scheme, and highlight the similarity between angioimmunoblastic TCL and PTCL-TFH. The findings show a group of cases with simultaneous expression of TFH markers and TH2 (GATA3), and indicate that ALK-negative ALCL is a heterogeneous condition. Cases of PTCL-NOS appear to be extremely heterogeneous, 


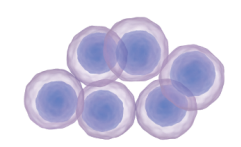

PTCL

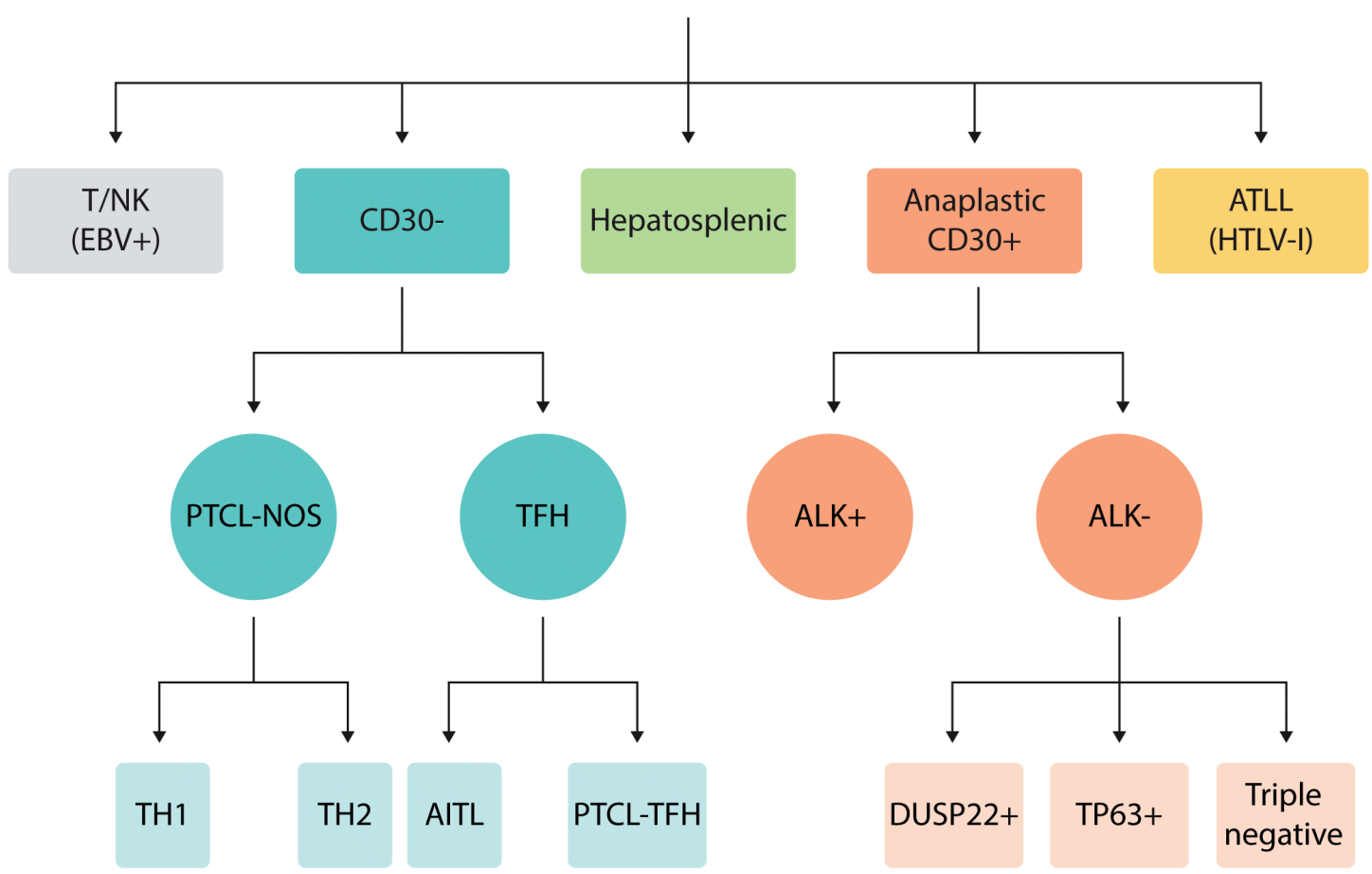

Figure 1. Peripheral T-cell lymphoma classification following the World Health Organization 2017 Lymphoma Classification, here restricted to the tumor types covered in the article. PTCL: Peripheral T-cell Lymphoma. NK: Natural Killer; ATLL: Adult T-cell Leukemia/Lymphoma; NOS: Not otherwise specified; TFH: T-Follicular Helper; ALK: Anaplastic Lymphoma Kinase; TH: T Helper; AITL: Angioimmunoblastic T-cell Lymphoma.

with the cases divided among the different phenotypes. In this study, ALK-negative ALCL had two distinct profiles, with or without expression of cytotoxic genes.

Clinical correlation confirmed the poor prognosis of PTCL (5-year OS=27\%), and the better prognosis for $\mathrm{ALK}^{+}$ and DUSP22+ ALCL, but failed to recognize markers that recognize groups with additional clinical variability. In particular, the division into TH1 and TH2 phenotypes in PTCL-NOS was not found to be clinically significant. It is of particular note that a $90 \%$ concordance was obtained between the three centers, thus emphasizing one of the stronger points in this approach, i.e. better reproducibility.

Although this is almost certainly not the last word on the process of creating molecular tools for the routine diagnosis of PTCL, it is an important step forward that establishes the viability of the proposal to integrate gene expression and variant recognition, and raises some questions about the PTCL subclasses as they are currently recognized.

Peripheral T-cell lymphoma diagnosis is an area in which molecular diagnosis can play an important role in the near future. Efforts in this field could prove even more relevant, with the provision, not only of diagnostic markers, but also of predictive therapeutic markers, by which the different lymphoma categories may be associated with the identification of useful targets that can be exploited for therapeutic purposes.

\section{Funding}

This work was supported by grants from the Instituto de Salud Carlos III (ISCIII) of the Spanish Ministry of Economy and Competence (MINECO, RTICC ISCIII and CIBERONC) (SAF2013-47416-R, RD06/002010107-RD012/0036/0060 and Plan Nacional I+D+I: PI17/2172, PI16/01294 and PIE15/0081), AECC, and the Madrid Autonomous Community.

\section{References}

1. Mak V, Hamm J, Chhanabhai M, et al. Survival of patients with peripheral T-cell lymphoma after first relapse or progression: spectrum of disease and rare long-term survivors. J Clin Oncol. 2013;31(16):1970-1976.

2. Federico M, Bellei M, Marcheselli L, et al. Peripheral T cell lymphoma, not otherwise specified (PTCL-NOS). A new prognostic model developed by the International $\mathrm{T}$ cell Project Network. Br J Haematol. 2018;181(6):760-769.

3. Weisenburger DD, Savage KJ, Harris NL, et al. Peripheral T-cell lymphoma, not otherwise specified: a report of 340 cases from the International Peripheral T-cell Lymphoma Project. Blood. 2011;117(12):3402-3408.

4. Laurent C, Baron M, Amara N, et al. Impact of Expert Pathologic Review of Lymphoma Diagnosis: Study of Patients From the French Lymphopath Network. J Clin Oncol. 2017;35(18):2008-2017.

5. de Leval L, Rickman DS, Thielen C, et al. The gene expression profile 
of nodal peripheral T-cell lymphoma demonstrates a molecular link between angioimmunoblastic T-cell lymphoma (AITL) and follicular helper T (TFH) cells. Blood. 2007;109(11):4952-4963.

6. Piccaluga PP, Agostinelli C, Califano A, et al. Gene expression analysis of peripheral T cell lymphoma, unspecified, reveals distinct profiles and new potential therapeutic targets. J Clin Invest. 2007;117(3):823-834

7. Cortes JR, Ambesi-Impiombato A, Couronne L, et al. RHOA G17V Induces T Follicular Helper Cell Specification and Promotes Lymphomagenesis. Cancer Cell. 2018;33(2):259-273.e7.
8. Heavican TB, Bouska A, Yu J, et al. Genetic drivers of oncogenic pathways in molecular subgroups of peripheral T-cell lymphoma. Blood. 2019;133(15):1664-1676.

9. Pedersen MB, Hamilton-Dutoit SJ, Bendix K, et al. DUSP22 and TP63 rearrangements predict outcome of ALK-negative anaplastic large cell lymphoma: a Danish cohort study. Blood. 2017;130(4):554-557.

10. Drieux F, Ruminy P, Abdel-Sater A, et al. Defining signatures of peripheral T-celllymphoma with a targeted 20-marker gene expression profiling assay.. Haematologica. 2020;105(6):1582-1592.

\title{
PIKing the next therapeutic target in multiple myeloma
}

\author{
Jessica L. Caro and Faith E. Davies \\ Perlmutter Cancer Center, NYU Langone Health, New York, NY, USA \\ E-mail: FAITH E DAVIES - faith.davies@nyulangone.org
}

doi:10.3324/haematol.2020.248971

$\Lambda$ rguably the most transformative myeloma therapies to date have been those which target essential processes involved in plasma cell function. Although their mechanism of action may not have been entirely obvious when first introduced, it has now become clear from cell-based studies that these therapies target protein degradation via the ubiquitin proteasome pathway, a critical process for plasma cell survival. Examples include proteasome inhibitors (such as bortezomib, carfilzomib, and ixazomib) and the immunomodulatory drugs (such as lenalidomide and pomalidomide), which inhibit the CUL4 E3 ubiquitin ligase, cereblon. Both drug classes are the 'go to' choices in current myeloma treatment. ${ }^{1}$ It therefore comes as no surprise that the search for other therapies targeting protein degradation pathways continues.

The key function of a normal plasma cell is to produce immunoglobulins. Studies have shown that myeloma plasma cells, which produce large quantities of $\mathrm{M}$ protein, are highly dependent on the multiple pathways that enable a cell to handle excess unfolded or misfolded proteins. Over the last 10 years, cancer researchers have explored many of these pathways with a view to therapeutic exploitation. The rationale is that inhibition of these pathways leads to a build up of unwanted or misfolded proteins, the induction of cellular stress, and ultimately to cancer cell death. Such pathways include not only the ubiquitin proteasome pathway but also the heat shock protein pathway, autophagy pathway, unfolded protein response pathway, and pathways involving lysosomes and aggresomes. ${ }^{2}$ However, translating in vitro findings into clinical success has been difficult. It has become clear that some cancer types are dependent on one pathway more than others, the pathways are interlinked, and the crosstalk between pathways enables the development of both primary and drug-induced mechanisms of resistance.

In this edition of Haematologica, Bonolo de Campos et al. describe a promising new approach for myeloma therapy by perturbing the autophagy and lysosome pathways. ${ }^{3}$ In autophagy, misfolded and aggregated proteins are sequestered in double-membraned vesicles called autophagosomes that eventually fuse with lysosomes for digestion and recycling. Previous studies have shown that myeloma cells require tight regulation of the autophagy pathway for cell survival, and genetic or therapeutic manipulation of this pathway induces growth inhibition and/or cell death. ${ }^{2,4,5}$

Phosphatidylinositol-3-phosphate 5 kinase (PIKfyve) is a phosphoinositide kinase with many diverse functions within the cell, including the generation of phosphorylated substrates critical to the regulation of autophagy. Inhibition of PIKfyve using the selective inhibitor apilimod has previously been investigated as a potential therapeutic approach for both inflammatory diseases and nonHodgkin lymphoma. ${ }^{7}$ Using an unbiased chemical screen, Bonolo de Campos et al. identified APY0201 and examined its activity along with that of apilimod and another novel PIKfyve inhibitor YM201636 in 25 human myeloma cell lines and 100 ex-vivo patient-derived samples. They confirmed dose-dependent inhibition of cell viability in all myeloma cell lines, with APY0201 being the most potent PIKfyve inhibitor. They additionally observed dosedependent sensitivities in $40 \%$ of ex-vivo patient-derived samples with APY0201. Mechanistic experiments suggested that exposure to APY0201 resulted in activation of the transcription factor EB (TFEB) leading to upregulation of autophagosome and lysosomal biogenesis. Exposure also disrupted lysosomal function leading to alterations in autophagic flux and a vacuolization phenotype.

As myeloma is a genetically and biologically heterogeneous disease, it is critical to identify which patients would benefit most from a new therapy. The prime example of the need for such an approach is venetoclax, a Bcl-2 inhibitor, which has been shown to be particularly efficacious in patients harboring a $\mathrm{t}(11 ; 14)$ translocation. ${ }^{8}$ Although targeting a pathway central to plasma cell survival should theoretically result in universal myeloma cell death, it has become clear that the genetic background of the cell influences response to therapy.9 For instance, whereas patients with a $t(14 ; 16)$ translocation tend to respond poorly to proteasome inhibitors, these therapies may be able to overcome some of the adverse outcome associated with the $t(4 ; 14)$ subgroup. ${ }^{10}$ Therefore, trying to incorporate genetic information into therapeutic decisionmaking may allow us to optimize treatment choices and response rates and to provide long-lasting remissions. Importantly, the authors have tried to assess this in their 